\title{
Engineering Exosome-Like Nanovesicles Derived from Asparagus cochinchinensis Can Inhibit the Proliferation of Hepatocellular Carcinoma Cells with Better Safety Profile
}

This article was published in the following Dove Press journal:

International Journal of Nanomedicine

\author{
Lei Zhang ${ }^{1} * *$ \\ Fengjun $\mathrm{He}^{1, *}$ \\ Lina Gao' \\ Minghui Cong' \\ Juan Sun ${ }^{2}$ \\ jialu $\mathrm{Xu}^{2}$ \\ Yutong Wang ${ }^{2}$ \\ Yang $\mathrm{Hu}^{\prime}$ \\ Sajid Asghar ${ }^{3}$ \\ Lihong $\mathrm{Hu}^{1,2}$ \\ Hongzhi Qiao',2,4 \\ 'Jiangsu Key Laboratory for Functional \\ Substances of Chinese Medicine, Nanjing \\ University of Chinese Medicine, Nanjing, \\ 210023, People's Republic of China; \\ ${ }^{2}$ State Key Laboratory Cultivation Base \\ for TCM Quality and Efficacy, School of \\ Pharmacy, Nanjing University of Chinese \\ Medicine, Nanjing, 210023, People's \\ Republic of China; ${ }^{3}$ Department of \\ Pharmaceutics, Faculty of Pharmaceutical \\ Sciences, Government College \\ University, Faisalabad, Pakistan; ${ }^{4}$ Jiangsu \\ Engineering Research Center for Efficient \\ Delivery System of TCM, Nanjing \\ University of Chinese Medicine, Nanjing, \\ 210023, People's Republic of China
}

*These authors contributed equally to this work

Correspondence: Hongzhi Qiao;

Lihong $\mathrm{Hu}$

School of Pharmacy, Nanjing University of

Chinese Medicine, I 38 Xianlin Avenue,

Nanjing, 210023, People's Republic of

China

Email qiaohz@njucm.edu.cn;

Ihhu@njucm.edu.cn
Background: Exosomes are a type of membrane vesicles secreted by living cells. Recent studies suggest exosome-like nanovesicles (ELNVs) from fruits and vegetables are involved in tissue renewal process and functional regulation against inflammatory diseases or cancers. However, there are few reports on ELNVs derived from medicinal plants.

Methods: ELNVs derived from Asparagus cochinchinensis (Lour.) Merr. (ACNVs) were isolated and characterized. Cytotoxicity, antiproliferative and apoptosis-inducing capacity of ACNVs against hepatoma carcinoma cell were assessed. The endocytosis mechanism of ACNVs was evaluated on Hep G2 cells in the presence of different endocytosis inhibitors. In vivo distribution of ACNVs was detected in healthy and tumor-bearing mice after scavenger receptors (SRs) blockade. PEG engineering of ACNVs was achieved through optimizing the pharmacokinetic profiles. In vivo antitumor activity and toxicity were evaluated in Hep G2 cell xenograft model.

Results: ACNVs were isolated and purified using a differential centrifugation method accompanied by sucrose gradient ultracentrifugation. The optimized ACNVs had an average size of about $119 \mathrm{~nm}$ and showed a typical cup-shaped nanostructure containing lipids, proteins, and RNAs. ACNVs were found to possess specific antitumor cell proliferation activity associated with an apoptosis-inducing pathway. ACNVs could be internalized into tumor cells mainly via phagocytosis, but they were quickly cleared once entering the blood. Blocking the SRs or PEGylation decoration prolonged the blood circulation time and increased the accumulation of ACNVs in tumor sites. In vivo antitumor results showed that PEGylated ACNVs could significantly inhibit tumor growth without side effects.

Conclusion: This study provides a promising functional nano platform derived from edible Asparagus cochinchinensis that can be used in antitumor therapy with negligible side effects.

Keywords: exosome, exosome-like nanovesicles, Asparagus cochinchinensis, engineering exosome, antitumor

\section{Introduction}

Extracellular vesicles (EVs) are a group of cell-derived membranous structures, which play an important role in intercellular communication and functional transduction. ${ }^{1,2}$ In recent years, mammalian cell-derived exosomes (a type of extracellular vesicle) have been widely used in the field of biomedicine, including diagnosis, regulation of disease progression and drug delivery. ${ }^{3,4}$ Similarly, plant cells also secrete exosome-like nanovesicles (ELNVs) that can participate in various physiological processes such as the 
inheritance of traits and defense response. ${ }^{5}$ However, plantderived ELNVs, especially from medicinal herbs, are scarcely reported for the biomedical application.

Medicinal plants have traditionally been utilized for improving human reproduction and fighting against diseases. ${ }^{6}$ Until today, medicinal plants are still an important avenue for the discovery of new drugs. ${ }^{7,8}$ Most of diseases (eg, malignant tumors) are multi-factor physiological abnormalities, thus single-drug administration does not yield the required clinical efficacy, while multi-drug combination is more likely to achieve synergistic effect with low toxicity. ${ }^{9}$ Unfortunately, to date, there is no ideal carrier that can pack and deliver multiple active ingredients to the target tissues. In recent years, ELNVs derived from edible fruits and vegetables have been studied. ${ }^{10}$ These ELNVs are composed of lipids, proteins and other natural components, and they have been found to possess similar or even better therapeutic benefits in comparison with the original plants. ${ }^{11}$ For example, oral delivery of ginger-derived ELNVs in mice has been reported to protect against alcohol-induced liver damage. ${ }^{12}$ It is expected the nano-scale ELNVs derived from medicinal plants would increase therapeutic outcomes in the treatment of refractory diseases such as cancers. ${ }^{13}$

Asparagus cochinchinensis (Lour.) Merr. (A. cochinchinensis) is a common edible plant that can also be used to make tea or wine. Besides, A. cochinchinensis has multiple pharmacological properties, including anticancer, antioxidation, antifungal, and antiaging activities. ${ }^{14-19}$ However, the effector mechanism remains partially understood. In this study, $A$. Cochinchinensis-derived nanovesicles (ACNVs) were isolated, and the antiproliferative activity and apoptosis-inducing mechanism against hepatocellular carcinoma (HCC) were investigated. Moreover, the in vivo distribution of ACNVs, the in vivo clearance mechanism, antitumor efficacy and safety evaluations were investigated in tumorbearing mice. The efforts are expected to facilitate the application of medicinal plant-derived NVs in the biomedical field.

\section{Materials and Methods}

\section{Materials}

Fresh A. cochinchinensis was purchased from standard cultivation base in Enshi of China and identified by Professor Yang $\mathrm{Hu}$, Department of Traditional Chinese Medicine Resources, Nanjing University of Chinese Medicine. 3-(4,5-dimethylthiazol-2-yl)-2,5-diphenyltetrazolium bromide (MTT) and distearoyl phosphoethanolamine-PEG2000 (DSPE-PEG) were obtained from Lipoid GmbH (Ludwigshafen, Germany). Formaldehyde (4\%), DAPI, DiR, PKH26, trypsin-EDTA solution, phosphate buffers (PBS), DMEM, and RPMI 1640 were supplied by KeyGEN BioTECH Co. Ltd (Nanjing, China). The primary antibodies of apoptosis-inducing factor (AIF), poly ADP-ribose polymerase (PARP), cleaved-PARP, and $\beta$ actin were purchased from Wuhan Sevier Biotechnology Co. (Wuhan, China). Ki67, proliferating cell nuclear antigen (PCNA), Bak, Bax, and caspase-9 were purchased from Abcam Co. (Shanghai, China). All secondary antibodies were purchased from Beyotime Biotechnology (Nantong, China). Annexin V-FITC/PI apoptosis kit was purchased from Fcmacs Biotechnology (Nanjing, China). HPLC-grade methanol $(\mathrm{MeOH})$, acetonitrile $(\mathrm{ACN})$, and $\mathrm{CHCl}_{3}$ were obtained from Merck (Darmstadt, Germany). Deionized (DI) water produced by a Milli-Q Plus System (Billerica, MA, USA) with a resistivity of $18.2 \mathrm{~m} \Omega \cdot \mathrm{cm}^{-1}$ was used in all the experiments. All other chemicals and reagents were obtained from Sigma Aldrich (Shanghai, China) and were used without further purification.

\section{Cells and Animals}

Human HCC cell lines (Hep G2, SMMC-7721, and Hep $3 \mathrm{~B})$ and normal hepatocyte cell line (LO 2) were obtained from American Type Culture Collection (ATCC), cultured at $37{ }^{\circ} \mathrm{C}$ under a humidified atmosphere of $5 \% \mathrm{CO}_{2}$ in DMEM (Hep G2, SMMC-7721, and LO 2) or 1640 (Hep 3B) medium supplemented with $10 \%$ fetal bovine serum, $100 \mathrm{IU} / \mathrm{mL}$ penicillin, and $100 \mu \mathrm{g} / \mathrm{mL}$ streptomycin.

Male BALB/c mice (18-22 g), BALB/c nude mice (18-22 $\mathrm{g}$ ), and Sprague-Dawley (SD) rats (200-250 g) were purchased from Nanjing Qing Longshan Animal Breeding Company (Nanjing, China). The animals were housed with ad libitum access to food and water at $25{ }^{\circ} \mathrm{C}$ and relative humidity of $55 \%$. Animal study protocols were approved by Animal Ethics Committee of Nanjing University of Chinese Medicine, and animal studies were performed in accordance with the Guide for Care and Use of Laboratory Animals.

\section{Preparation of ACNVs}

ACNVs were isolated from the fresh juice of A. cochinchinensis through differential centrifugation. ${ }^{20}$ Briefly, the roots of $A$. cochinchinensis were carefully washed with DI water and manually squeezed. The obtained juice was sequentially centrifuged at $4000 \mathrm{~g}$ for $60 \mathrm{~min}, 10,000 \mathrm{~g}$ for 60 $\mathrm{min}$ to remove large particles and fibres. The supernatant was then centrifuged at $120,000 \mathrm{~g}$ for $120 \mathrm{~min}$ in a Type $32 \mathrm{Ti}$, flatangle rotor; the pellet was suspended in $2 \mathrm{~mL}$ ice-cold PBS, 
transferred to a gradient sucrose solution $(8,30,45$ and $60 \%$ (g/ $\mathrm{v})$ ), and ultracentrifuged at $120,000 \mathrm{~g}$ for another $60 \mathrm{~min}$. The ACNVs (band at the $30 \% / 45 \%$ interface) were collected, suspended in $2 \mathrm{~mL}$ ice-cold PBS, and ultracentrifuged at $120,000 \mathrm{~g}$ for $60 \mathrm{~min}$. Afterwards, the pellets were collected and dried under nitrogen atmosphere for quantification.

\section{Characteristics of ACNVs}

The particle size and zeta potential of ACNVs crude extracts were measured by dynamic light scattering (DLS) technique using a Zetasizer (Malvern Instruments, Worcestershire, UK) at $25{ }^{\circ} \mathrm{C}$ with a scattering angle of $90^{\circ}$. Particle diameter and concentration were measured by Nanoparticle Tracking Analysis (NTA) (Nanosight NS300, Malvern Instruments, Worcestershire, UK). Briefly, samples were diluted to $10-20 \mu \mathrm{g} / \mathrm{mL}$ in $\mathrm{PBS}$, and 3 videos of $30 \mathrm{~s}$ were acquired in triplicate.

The surface morphology of each sucrose-gradient band was observed using transmission electron microscopy (TEM) (HITACHI, HT-7800, Tokyo, Japan). Briefly, 10 $\mu \mathrm{L}$ sample was deposited on a carbon-coated support copper grid, negative stained with $10 \mu \mathrm{L} 1 \%$ phosphotungstic acid for 2.5 min followed by air dry and then examined under TEM.

\section{Composition Analysis of ACNVs}

The total lipids of ACNVs were extracted according to the previously reported method. ${ }^{21}$ A mixed solvent of chloroform/ methanol (2:1) was added to ACNVs suspension. The organic phase was collected, dried, and resuspended in chloroform. The lipid samples were separated on a silica gel thin-layer chromatography (TLC) plate using a mixture of chloroform/ methanol/acetic acid (190:9:1) as a developing solvent and visualized with $10 \% \mathrm{CuSO}_{4}$ in $8 \%$ phosphoric acid reagent. ${ }^{22}$ ACNVs were resuspended in PBS and treated with RIPA lysis buffer $(1: 5, \mathrm{v} / \mathrm{v})$ (Beyotime, Nantong, China) for $5 \mathrm{~min}$ on icebath, thereafter lysates were centrifuged $(12,000 \mathrm{~g}, 5 \mathrm{~min})$ at $4^{\circ}$ C. Ten-microliter proteins in the supernatant were loaded and severed by $12 \%$ SDS-PAGE and stained with coomassie blue staining for $1 \mathrm{~h}$. ACNVs were resuspended in ice-cold PBS with $10 \mu \mathrm{g} / \mathrm{mL}$ of RNase A and incubated at $37^{\circ} \mathrm{C}$ for $2 \mathrm{~h}$, and then washed with PBS and pelleted using ultracentrifugation (120 000g, $60 \mathrm{~min}$ ). The RNA-depleted ACNVs were subjected to RNA extraction to evaluate the depletion efficiency. ${ }^{13}$

\section{In vitro Antitumor Activity}

Cell viability was assessed by MTT assay as previously described. $^{23}$ Briefly, cell suspensions of Hep G2, Hep 3B,
SMMC-7721, and LO 2 were inoculated into 96-well plates at a density of $5 \times 10^{4}$ cells/well and incubated at $37{ }^{\circ} \mathrm{C}$ overnight. The culture medium was discarded, and $200 \mu \mathrm{L}$ ACNVs with different concentrations were added for 24 $\mathrm{h}$ incubation at $37^{\circ} \mathrm{C}$. Then, $20 \mu \mathrm{L}$ MTT solution $(5 \mathrm{mg} / \mathrm{mL})$ was added and incubated for another $4 \mathrm{~h}$. The supernatant was discarded, and $150 \mu \mathrm{L}$ DMSO was added to dissolve bluepurple armor crystals. The optical density (OD) was read at 490 nm using a microplate reader (Tecan Infinite 200 PRO, Männedorf, Switzerland). Cell viability (\%) was calculated as the ratio of OD in test samples to OD in control samples.

Annexin V-FITC/PI apoptosis kit was used to detect tumor cell apoptosis. Briefly, Hep G2 cells were inoculated into 12well plates at a density of $1 \times 10^{6}$ cells/plate, incubated at $37^{\circ} \mathrm{C}$ overnight, and treated with different concentrations of ACNVs for $24 \mathrm{~h}$. Cell apoptosis was measured using FACS Calibur flow cytometer (BD Biosciences, San Jose, CA) according to standard kit protocol and analyzed by FlowJo software (Tree Star, Ashland OR).

The proliferation and apoptosis-related protein expression were analyzed by Western blot. Hep G2 cells were treated with ACNVs for $24 \mathrm{~h}$. Then, the total proteins were collected from each group. Proteins were resolved on $10 \%$ SDS-PAGE and electroblotted onto nitrocellulose membranes. Membranes were blocked with $2.5 \%$ milk solution, followed by overnight incubation at $4{ }^{\circ} \mathrm{C}$ with primary antibodies. The blots were then incubated with horseradish peroxidase (HRP)-coupled secondary antibody. The primary antibodies were as following: Ki67, PCNA, BAX, BAK, AIF, caspase-9, PARP, cleaved-PARP, and $\beta$-actin.

\section{ACNVs Labeling for in vitro and in vivo Tracking}

Thirty-milligram ACNVs in $1 \mathrm{~mL}$ PBS were incubated with PKH 26, DiR or $\mathrm{DiO}$ at a final concentration of 100 $\mathrm{ng} / \mathrm{mL}$ of dye for $30 \mathrm{~min}$ at $37^{\circ} \mathrm{C}$. The unencapsulated dye was removed by ultrafiltration using $100 \mathrm{kDa}$ filter membrane (Merck Millipore, Burlington, Massachusetts).

\section{ACNVs Uptake Assessment}

Hep G2 or LO 2 cells seeded in 12-well plates $\left(1 \times 10^{6}\right.$ cells/plate) were incubated with PKH 26-labeled ACNVs $(30 \mu \mathrm{g} / \mathrm{mL}$ ) for $0.5,1,2$, and $4 \mathrm{~h}$. Afterward, the cells were washed with cold PBS, trypsinized, resuspended in $0.5 \mathrm{~mL}$ PBS, and analyzed using a flow cytometer. To evaluate the potential endocytic pathways of ACNV, Hep G2 cells were cultured at $37{ }^{\circ} \mathrm{C}$ in the presence of different endocytosis 
inhibitors (amiloride $150 \mu \mathrm{g} / \mathrm{mL}$, nystatin $0.5 \mu \mathrm{g} / \mathrm{mL}$, chlorpromazine hydrochloride $10 \mu \mathrm{g} / \mathrm{mL}$ or cytochalasin D $25 \mu \mathrm{g} / \mathrm{mL}$ ) for $2 \mathrm{~h}$ followed by the addition of DiOlabeled ACNVs. After incubation for another $4 \mathrm{~h}$, cells were fixed for fluorescence imaging and quantification by FACS analysis.

\section{In vivo Distribution of $A C N V s$ in Tumor-Free and Tumor-Bearing Mice After SRs Blockade}

Hep G2 cell suspensions were subcutaneously inoculated into the right hind leg of BALB/c nude mice, and the tumor size was monitored using a vernier caliper. Tumor volume $(\mathrm{V})$ was calculated as $\mathrm{V}=\mathrm{L} \times \mathrm{W}^{2} / 2$, where $\mathrm{L}$ and $\mathrm{W}$ are the length and width of the tumor, respectively.

Normal BALB/c mice and Hep G2 cell xenograft model in $\mathrm{BALB} / \mathrm{c}$ nude mice were intravenously injected via the tail vein with DiR-labeled ACNVs (15 mg per mouse). For in vivo SRs blockage study, mice were injected with the SRs inhibitor dextran sulfate at a dose of $30 \mathrm{mg} / \mathrm{kg}, 2 \mathrm{~h}$ prior to ACNVs injection. ${ }^{24}$ Control mice received $100 \mu \mathrm{L}$ saline. At predetermined time intervals, the animals were anaesthetized with $2 \%$ isoflurane and imaged under an IVIS Lumina imaging system (PerkinElmer, Waltham, MA) at $\lambda_{\mathrm{ex}} / \lambda_{\mathrm{em}}=740 \mathrm{~nm} / 790 \mathrm{~nm}$. At $24 \mathrm{~h}$ post-administration, mice were sacrificed; blood, tumor tissues, and the main organs were collected for ex vivo imaging. Region of interests (ROIs) were circled around the organs, and the fluorescence intensity was analyzed using Living Image Software 4.5.2.

\section{PEG Engineering of ACNVs}

To improve the pharmacokinetic profile of ACNVs, PEG engineering process was operated by incorporating DSPEPEG into lipid membrane of ACNVs to obtain PEGACNVs. In brief, DSPE-PEG $(1,2,3,4$ or $5 \mathrm{mg})$ was added into $1 \mathrm{~mL}$ ACNVs $(30 \mathrm{mg} / \mathrm{mL})$ followed by vortex and ultrasound for $20 \mathrm{~min}$ under ice-bath. The mixture was purified by five cycle ultrafiltration using $100 \mathrm{kDa}$ filter membrane to remove free DSPE-PEG. The degree of PEGylation was optimized by the pharmacokinetic profile. Briefly, SD rats were intravenously injected with PKH 26labeled PEG-ACNVs at the dose of $100 \mathrm{mg} / \mathrm{kg}$. Retroorbital blood was collected at $0.25,0.5,1,2,4,8,12$, and $24 \mathrm{~h}$ post-injection; $50 \mu \mathrm{L}$ whole blood was taken in 96-well plates and imaged using IVIS Lumina imaging system. The particle size and zeta potential of PEGylated
ACNVs were measured by Zetasizer, and the morphology was observed using TEM.

\section{In vivo Antitumor Activity and Toxicity Evaluation}

When the tumor size reached $100-200 \mathrm{~mm}^{3}$, the mice were randomly divided into three groups, and they received intravenous injection of saline, ACNVs or PEG-ACNVs at the dose of $200 \mathrm{mg} / \mathrm{kg}$ every other day for a total of seven doses. The tumor size and body weight were regularly monitored. On day 14, mice were euthanized, and the tumor was harvested, washed with saline thrice, and then fixed in $4 \%$ polyformaldehyde for hematoxylin and eosin (H\&E) staining and TUNEL apoptosis staining. Furthermore, main organs were also collected, and H\&E staining of tissues was subsequently performed. Blood samples were used for biochemistry assay and routine blood test, including aspartate aminotransferase (AST), alanine aminotransferase (ALT), blood urea nitrogen (BUN), creatinine (CRE), counting of white blood cells (WBC), and total bilirubin (TBIL).

\section{Statistical Analysis}

Data are presented as mean \pm standard deviation. Statistical analysis was performed using analysis of variance, followed by Bonferroni post hoc correction, and $p<0.05$ was used as the minimal level of statistical significance.

\section{Results and Discussion \\ Preparation, Characterization and Composition Analysis of ACNVs}

ACNVs crude extracts were isolated using a classic differential centrifugation method. After multi-step centrifugation process, the particle size distribution of ACNVs crude extracts was narrowed, and the average particle size was reduced to about $190 \mathrm{~nm}$ (DLS data) (Figure 1A). Three main bands were formed after sucrose gradient ultracentrifugation (Figure 1B). The average size was about $92 \mathrm{~nm}$ for NVs found in band 1, $119 \mathrm{~nm}$ from band 2, and $179 \mathrm{~nm}$ from band 3 (NTA data) (Figure 1C). The majority of ACNVs accumulated at the 30\%/ $45 \%$ interface (band 2) and showed a typical cup-shaped nanostructure, which was consistent with previous reports. ${ }^{25}$ The regularity and uniformity of NVs in band 2 were better than those in other bands (Figure 1C). ACNVs in band 2 had a negative zeta potential value of $-21 \mathrm{mV}$.

TLC analysis showed that a variety of lipids were present in ACNVs (Figure 1D). The proteins from ACNVs were in a range of $20-250 \mathrm{kDa}$ (Figure 1E). The RNAs in ACNVs 
A

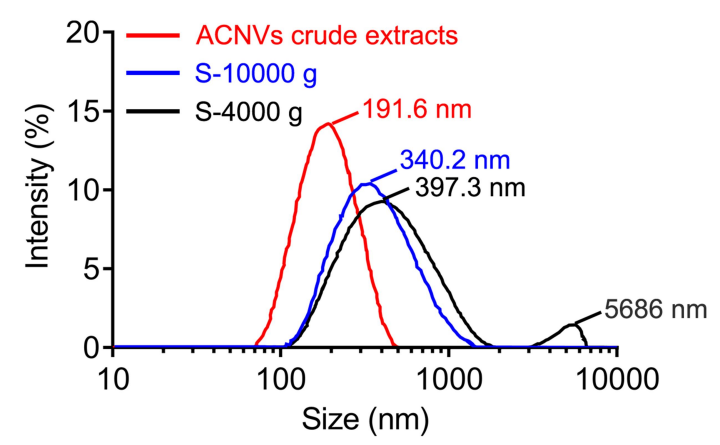

D

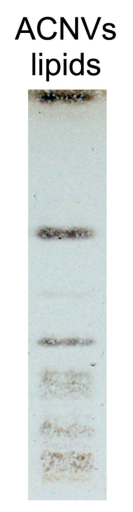

$\mathbf{E}$

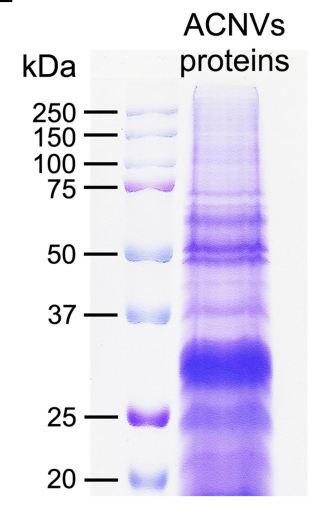

B

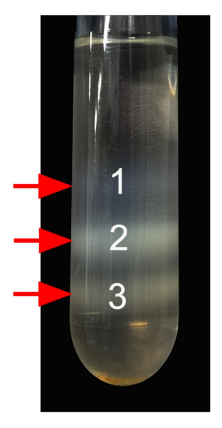

C
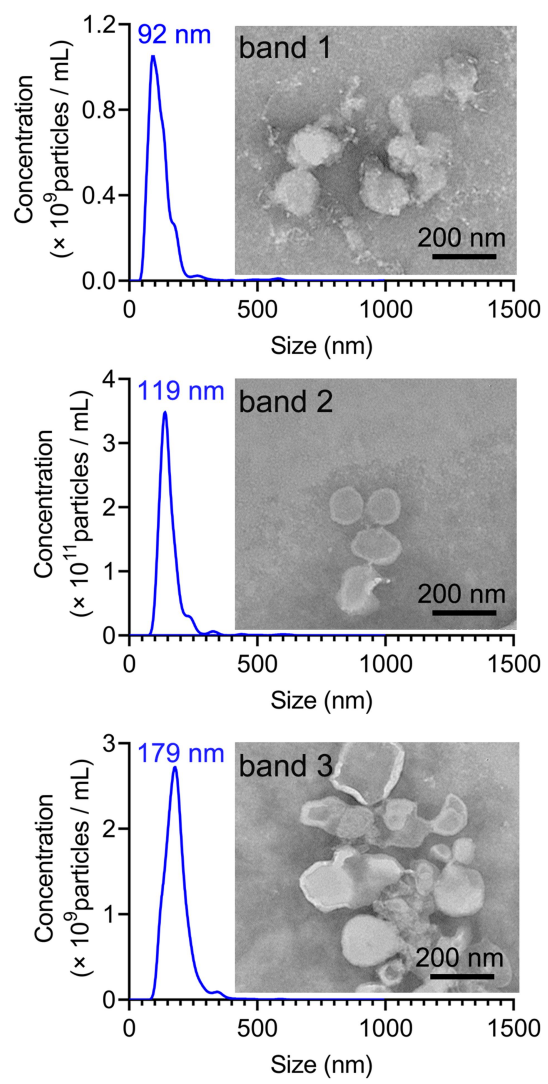

Figure I Preparation, characterization and composition analysis of ACNVs. (A) Size distribution changes during the isolation process as visualized by DLS. S-4000 $\mathrm{g}$ and $\mathrm{S}-10,000 \mathrm{~g}$ was the supernatant after centrifugation at $4000 \mathrm{~g}$ and $10,000 \mathrm{~g}$, respectively. (B) Three bands formed after sucrose gradient ultracentrifugation. (C) The size and morphology of each sucrose-gradient band was characterized by NTA and TEM, respectively. Scale bar $=200 \mathrm{~nm}$. (D) TLC analysis of ACNVs lipids. The lipids from ACNVs were separated on a TLC silica gel plate and visualized with $\mathrm{CuSO}_{4}$ phosphoric acid solution. (E) Protein gel of ACNVs proteins. The proteins extracted from ACNVs were separated by SDS-PAGE and stained with Coomassie blue staining. (F) RNA gel of ACNVs RNAs. The RNAs extracted from ACNVs were treated with or without RNase and run on a $1 \%$ agarose gel. The RNA ladder of $100-5000$ nucleotides (nt) was used.

were primarily small-sized RNAs (Figure 1F). RNase treatment led to complete degradation of the nucleic acids extracted from ACNVs, indicating that they were mainly RNAs. ${ }^{22}$ Thus, the purified ACNVs were verified as nano-scaled structures containing lipids, proteins, and RNAs.

\section{In vitro Antitumor Activity}

The in vitro cytotoxicity of ACNVs against human HCC cell lines (Hep G2, Hep 3B, and SMMC-7721) and normal hepatocyte cell line (LO 2) was evaluated by using MTT assay. As shown in Figure 2A, the growth of various HCC cells was significantly inhibited by the ACNVs to a varying extent. The half-inhibitory concentration $\left(\mathrm{IC}_{50}\right)$ of ACNVS against Hep G2, Hep 3B or SMMC-7721 cells was $42.5,31.7$ or $37.8 \mu \mathrm{g} /$ $\mathrm{mL}$, respectively. In contrast, ACNVs displayed negligible cytotoxicity against LO 2 cells $\left(\mathrm{IC}_{50} 279.8 \mu \mathrm{g} / \mathrm{mL}\right)$, indicating the tumor-selective inhibition activity. Similar results were reported that Asparagus polysaccharide can induce approximately $80 \%$ growth inhibition of HCC cells in comparison to $20 \%$ inhibition of LO 2 cells. ${ }^{14}$ Hep G 2 cell is one of the most widely used cells in the anti-tumor evaluation of Asparagus extracts. ${ }^{16,26}$ To obtain more information for comparison, Hep G2 cell line was selected for the subsequent experiments. To further confirm the effects of ACNVs on the proliferation of neoplastic cells, the expression levels of Ki67 and PCNA were tested by Western blotting. The results showed the level of Ki67 and PCNA were significantly decreased in a dose-dependent manner (Figure S1a and b), which confirmed that ACNVs could inhibit the proliferation of Hep G2 cells.

Apoptosis assay of ACNVs was also performed on Hep G2 cells by flow cytometry. As displayed in Figure 2B, after 24 $\mathrm{h}$ incubation, ACNVs showed a dose-dependent apoptosisinducing activity. At the concentration of $40 \mu \mathrm{g} / \mathrm{mL}$, ACNVs led to an apoptosis ratio of $46.5 \%$. Furthermore, the expression of apoptosis-associated factors was evaluated. As shown in Figure 2C, ACNVs treatment caused a dose-dependent 

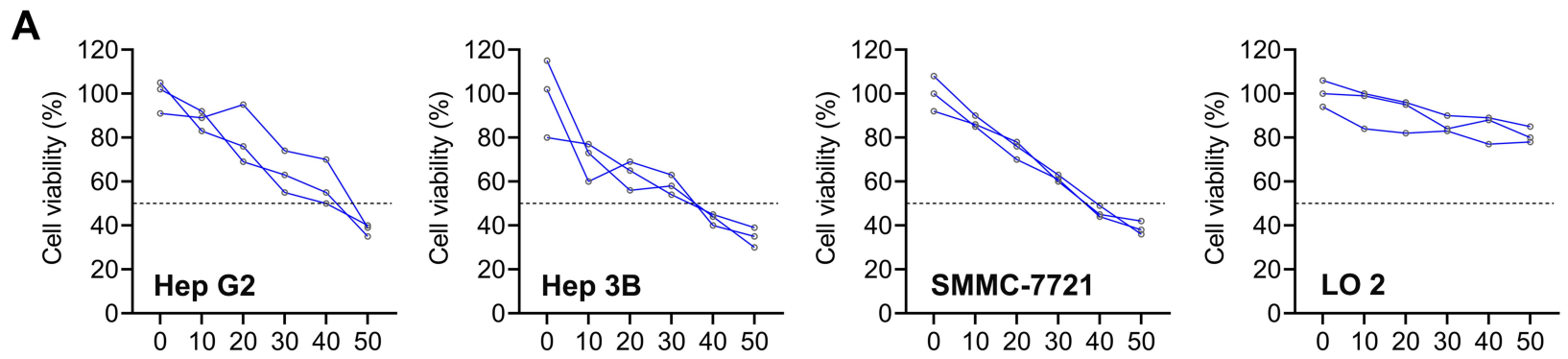

Concentration of ACNVs $(\mu \mathrm{g} / \mathrm{mL})$ Concentration of ACNVs $(\mu \mathrm{g} / \mathrm{mL})$ Concentration of ACNVs $(\mu \mathrm{g} / \mathrm{mL})$ Concentration of $A C N V s(\mu \mathrm{g} / \mathrm{mL})$

B

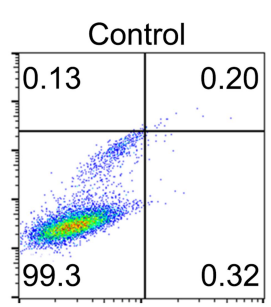

$30 \mu \mathrm{g} / \mathrm{mL}$

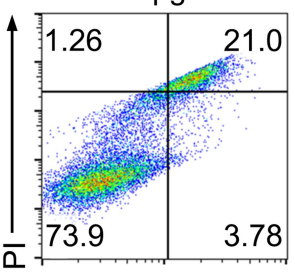

Annexin V-FITC

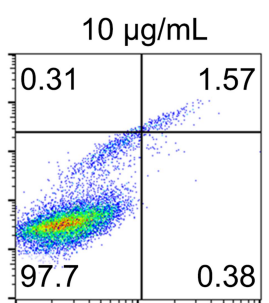

$40 \mu \mathrm{g} / \mathrm{mL}$

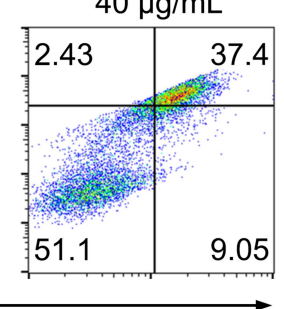

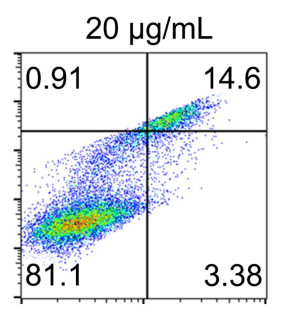

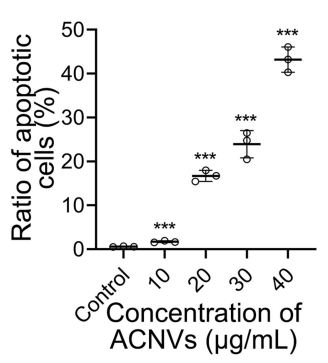

C Conc. $(\mu \mathrm{g} / \mathrm{mL})$

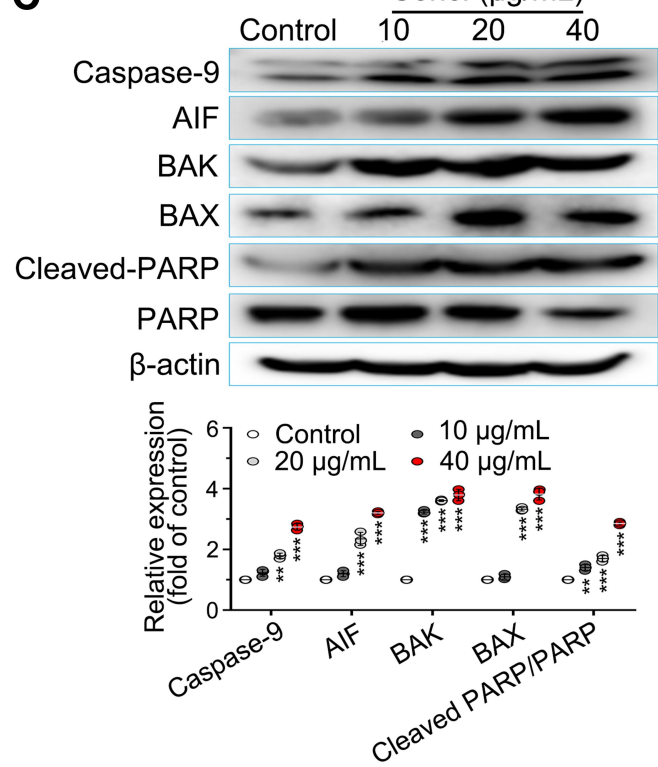

Figure 2 Cytotoxicity and apoptosis-inducing activity of ACNVs. (A) Cytotoxicity of ACNVs on different cells detected by MTT method. Cell viability of $50 \%$ was marked by dotted lines. The half-inhibitory concentration $\left(I_{50}\right)$ of ACNVS on Hep G2, Hep 3B, SMMC-772I or LO 2 cells was $42.5,31.7,37.8$ or $279.8 \mu g / m L$, respectively. (B) Flow cytometric analysis of Hep G2 cell apoptosis induced by ACNVs with different concentrations for $24 \mathrm{~h}$ by using Annexin V-FITC/PI staining. (C) Immunoblotting for apoptosis-related proteins of Hep G2 cells treated with ACNVs. ${ }^{* *} p<0.01$, $* * * p<0.001$ vs control.

increase in the protein levels of AIF, Bax, and Bak to trigger activation of caspase-9, which can systematically cleave key cellular proteins including DNA repair enzyme PARP and ultimately lead to cell death. ${ }^{27}$ The above results suggested that the cytotoxicity of ACNVs against tumor cells may be partially associated with the apoptosis-inducing mechanism. Accumulating data indicate ELNVs carry a large number and variety of natural molecules, ${ }^{28}$ which can simultaneously target to multiple pathogenic factors to inhibit the tumor growth. ${ }^{29}$ Therefore, the detailed mechanisms of specific components need to be further explored.

\section{Cellular Uptake Assessment}

Cellular uptake of ACNVs was measured by a flow cytometer. As shown in Figure 3A and B, a time-dependent internalization of ACNVs was observed on both Hep G2 and LO2 cells. Comparing to the LO2 cells, the uptake of ACNVs by Hep G2 cells appeared more efficient. After $4 \mathrm{~h}$ incubation, the fluorescence intensity of Hep G2 cells was almost twice than that of LO 2 cells, which may partially account for the difference in the cytotoxicity of ACNVs against two types of cells. To date, there is no conclusive evidence to explain the differences in ACNVs uptake by different cells. Based on the literatures, we hypothesized that this may be related to differences in the composition and properties of cell membranes. ${ }^{30-32}$ In addition, the specific integrin proteins of ACNVs may also determine cell tropism. ${ }^{11,33,34}$

To further investigate the endocytosis mechanism of ACNVs, HepG2 cells were incubated with endocytosis inhibitors (chlorpromazine hydrochloride, amiloride, nystatin, and cytochalasin D). ${ }^{35}$ As shown in Figure $3 \mathrm{C}$ and D, the uptake of DiO-ACNVs was significantly inhibited by cytochalasin $\mathrm{D}$ (inhibition rate of $68 \%$ ), an inhibitor of actin polymerization required for phagocytosis. However, chlorpromazine 
A

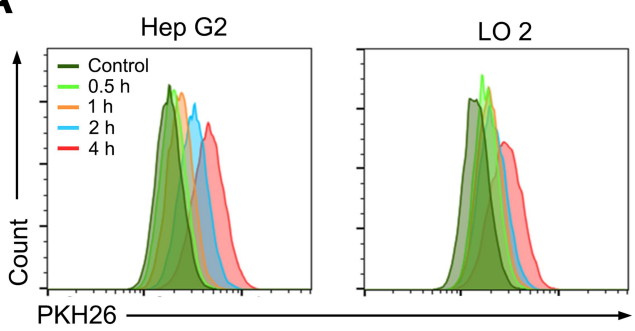

C

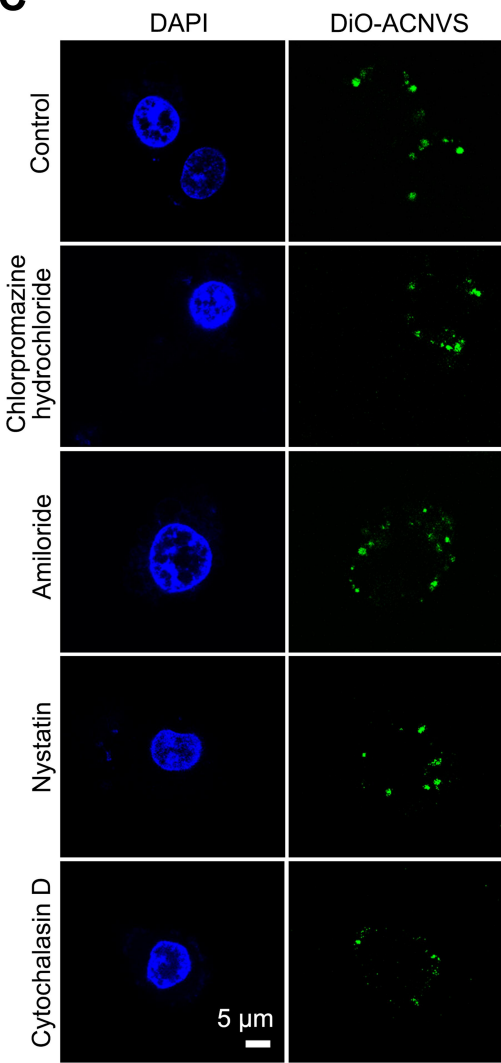

B

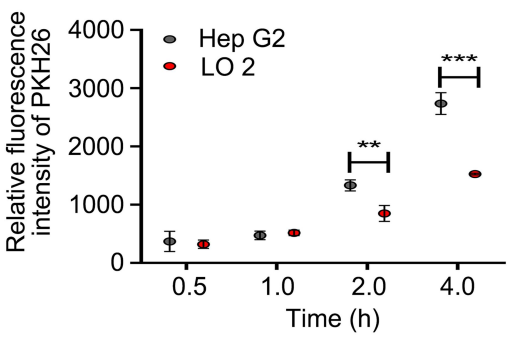

D
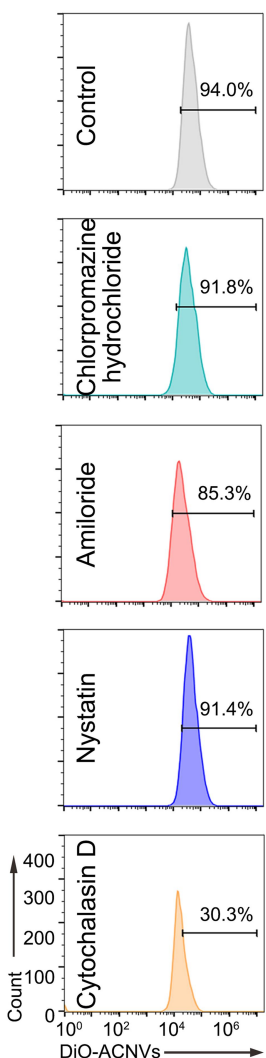

Figure 3 Cellular uptake of ACNVs. (A) Intracellular fluorescence intensity of Hep G2 cells treated with ACNVs for different periods. (B) Quantification of cellular uptake after incubation with ACNVs. (C) Potential endocytosis pathways of DiO-labeled ACNVs on Hep G2 cells. (D) Uptake efficiency of DiO-labeled ACNVs quantified by flow cytometry. ${ }^{* *} p<0.01$, ${ }^{* * *} p<0.001$.

hydrochloride (an inhibitor of clathrin-mediated endocytosis), amiloride (an inhibitor of macropinocytosis), and nystatin (an inhibitor of caveolae-medicated endocytosis) had slight effects on the uptake of DiO-ACNVs (less than 10\% inhibition rate), suggesting that ACNVs were most possibly internalized via phagocytosis pathway. ${ }^{35}$

\section{In vivo Distribution of $A C N V s$}

To determine the tissue tropism of ACNVs, in vivo biodistribution of DiR-labeled ACNVs was evaluated in mice using a noninvasive imaging system. The preliminary experiment showed that fluorescence signals in mice post intravenous injection of ACNVs were mainly located in the livers and spleens, which was in accordance with the in vivo manner of the common nano-scaled particles. ${ }^{36}$ The liver and spleen are known as the main units of the mononuclear phagocyte system (MPS). Systemically delivered naked nanoparticles are easily cleared by MPS, resulting in minimal accumulation within the target area, which is a major hurdle for the nanocarrier-based therapeutic approaches. ${ }^{37}$ The elevated fluorescence signals in the liver and spleen implied that the nano-sized ACNVs had the same results.

It was reported that depletion of monocytes/macrophages could significantly prolong the systemic half-life of 
A

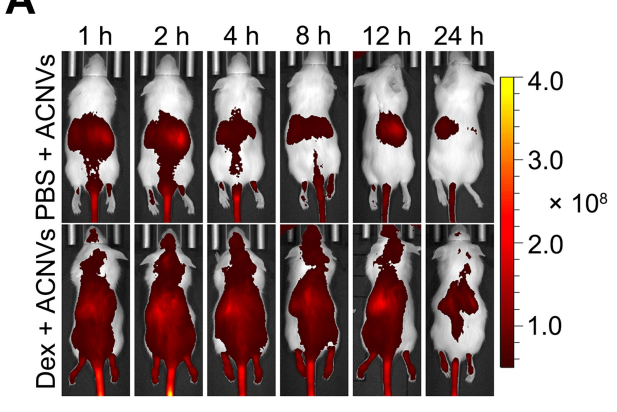

C

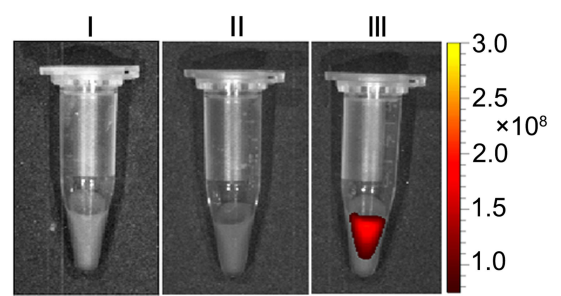

B

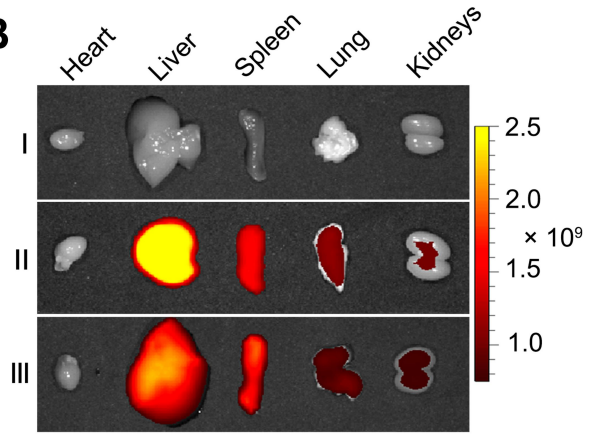

D

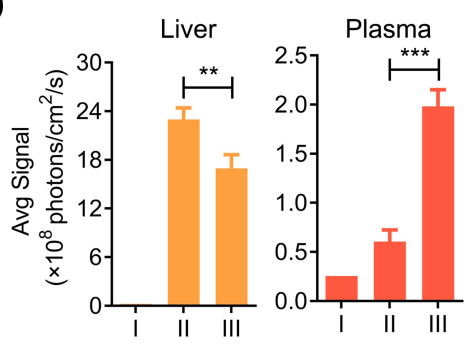

Figure 4 In vivo and ex vivo fluorescence imaging of ACNVs in BALB/c mice. (A) In vivo fluorescence images of Hep G2 tumor-bearing mice captured at different intervals post intravenous injection of DiR-labeled ACNVs. SRs blockade by dextran sulfate (Dex) pretreatment decreases liver clearance of ACNVs. (B) Ex vivo fluorescence images of tissues collected from mice injected with saline (I), ACNVs (II) or Dex and ACNVs (III) after $24 \mathrm{~h}$. (C) Ex vivo fluorescence images of blood samples collected from mice with different treatments. (D) Semiquantitative relative biodistribution of ACNVs in liver and plasma, determined by the fluorescence intensities obtained from b and c. $* * p<$ 0.01 , $* * * p<0.001$.

EVs in mice, which implied that phagocytosis by monocytes/macrophages is an important clearance mechanism of EVs in vivo. ${ }^{38,39} \mathrm{SRs}$ is a class of transmembrane proteins widely expressed on macrophages, which are responsible to facilitate the uptake of a wide variety of ligands ranging from foreign substances to endogenous lipids/proteins, especially negatively charged molecules. ${ }^{40}$ Inspired by the tendency of SRs, the in vivo behavior of negatively charged ACNVs was investigated in SRs-exhausted mice by using a well-known inhibitor dextran sulfate.

Normal BALB/c mice were injected with DiR-labeled ACNVs or dextran sulfate $(30 \mathrm{mg} / \mathrm{kg}) 2 \mathrm{~h}$ prior to ACNVs injection, and the fluorescent images were captured at predetermined time points (Figure 4A). At $24 \mathrm{~h}$ postadministration, in comparison to the control, the fluorescence signal of livers isolated from dextran sulfate treated mice was significantly suppressed (Figure 4B and D). Moreover, blood samples from mice treated with dextran sulfate exhibited about a 3 -fold increase than the normal group (Figure 4C and D). The above results indicated that SRs were involved in the clearance of ACNVs, and the blockage of SRs could lower the blood clearance rate of ACNVs and reduce the accumulation in livers. Similar conclusion was obtained in HepG2 tumor xenograft nude mice (Figure $\mathrm{S} 2 \mathrm{a}-\mathrm{c}$ ). An increased intratumoral ACNVs accumulation could be observed in mice pretreated with dextran sulfate. We speculate that the cascade results are mainly attributed to the enhanced permeability and retention (EPR) effect in tumors. ${ }^{41}$

\section{Preparation and in vivo Distribution of PEG-ACNVs}

To decrease the non-specific clearance of ACNVs, PEGylated ACNVs were prepared to improve the in vivo pharmacokinetics, tumor targeting, and pharmacodynamic properties. ${ }^{42}$ The modification degree of PEG was optimized by the in vivo pharmacokinetic profiles of PEG-ACNVs (Figure S3a and b). Results showed PEG 2 (6.7 wt.\% DSPE-PEG) had the highest blood accumulation than other groups, therefore the follow-up studies were carried out with the optimal PEG 2 formulation, named as PEG-ACNVs. The size and zeta potential of PEG-ACNVs were $108 \pm 17 \mathrm{~nm}$ and $-10 \mathrm{mV}$, respectively. The TEM image showed PEG-ACNVs retained the typical cup-shaped morphology (Figure S4).

The targeting ability of PEG-ACNVs was determined by in vivo imaging in HepG2 tumor xenograft nude mice. As shown in Figure 5A, a significantly increased fluorescence signal could be detected in tumor tissues from mice treated with PEG-ACNVs compared to the ACNVs group. In parallel, blood retention period of PEG-ACNVs was obviously 
A

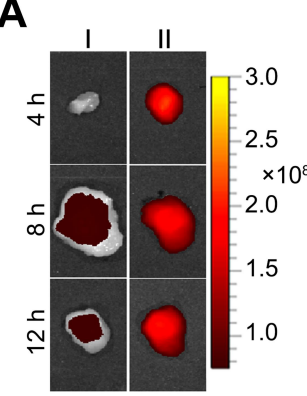

B

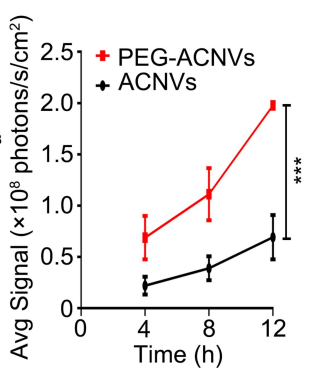

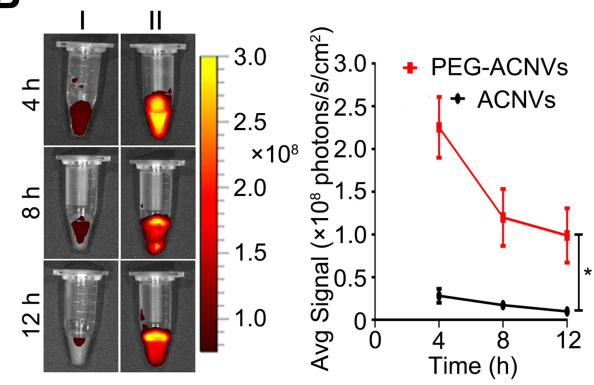

Figure 5 Influence of PEGylation on in vivo distribution of ACNVs in Hep G2 tumor xenograft nude mice. (A) Ex vivo fluorescence imaging and quantification of tumors from mice treated with ACNVs ( I ) and PEG-ACNVs (II). (B) Ex vivo fluorescence imaging and quantification of blood samples from mice treated with ACNVs ( I ) and PEG-ACNVs (II). ${ }^{*} p<0.05, * * * p 0.001$.

prolonged (Figure 5B), which was attributed to the escape of nanovesicles treated by PEGylation from the MPS. ${ }^{42}$

\section{In vivo Antitumor Activity and Toxicity Evaluation}

The antitumor efficacy of PEG-ACNVs was evaluated in HepG2 tumor xenograft nude mice. During the efficacy test, no noticeable abnormality was observed in any study group except for the weight differences in the animals
(Figure 6A). The weight loss observed in mice treated with ACNVs may be related to direct exposure of ACNVs to blood, but the underlying mechanism is not clear. As shown in Figure 6B-D, the tumor growth was significantly suppressed after successive intravenous injections of ACNVs and PEG-ACNVs compared to the negative control. The mice receiving $\mathrm{PEG}-\mathrm{ACNV}$ s had a stronger tumorinhibiting capacity than ACNVs group, which was attributed to the probably prolonged blood accumulation and increased
A

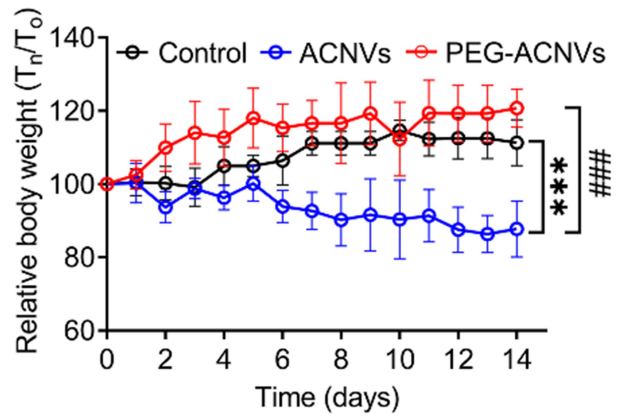

D

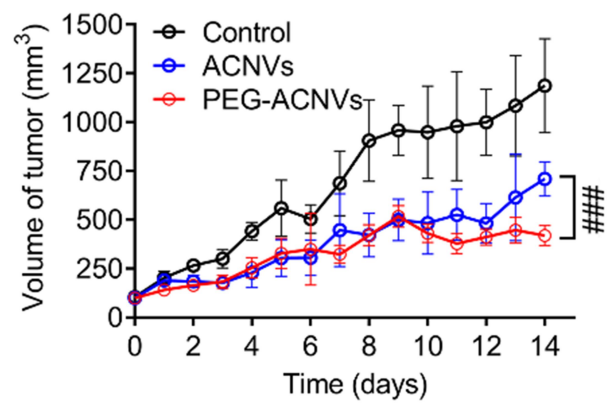

B

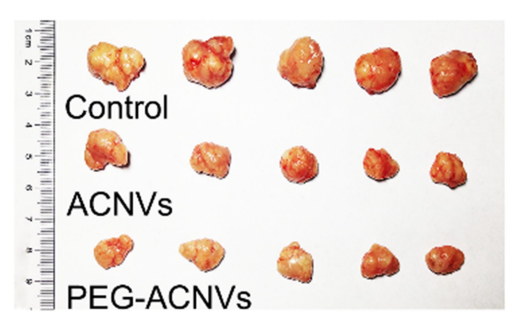

$\mathbf{E}$

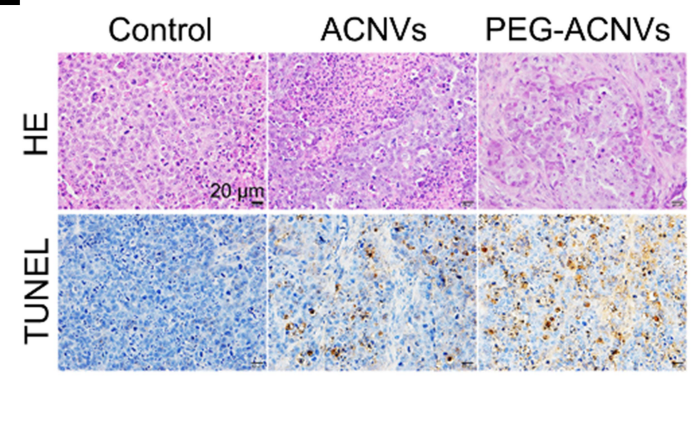

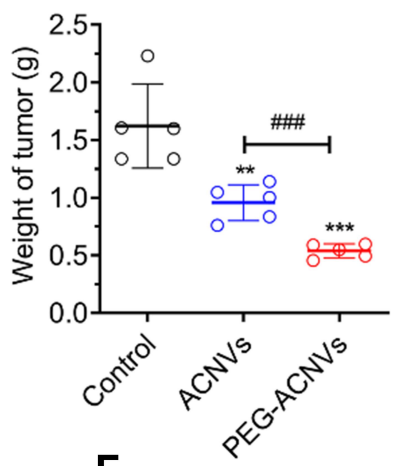

$\mathbf{F}$

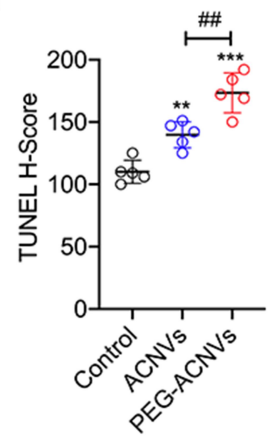

Figure 6 In vivo antitumor efficacy in Hep G2 tumor xenograft nude mice $(n=5)$. (A) Body weight changes during treatments. (B) Representative images of tumors in mice after different treatments. (C) Weight of tumors from mice after different treatments. (D) Volume of tumors from mice after different treatments. (E) Histological and apoptosis comparison after different treatments, scale bar $=20 \mu \mathrm{m}$. (F) Quantitative comparison of the positive staining of TUNEL cell numbers in tumor tissues of each group. ${ }^{\#} p<0.01,{ }^{\# \#} p<0.001$ vs ACNVs group. ${ }^{* *} p<0.01$, ***p $<0.001$ vs control. 
A

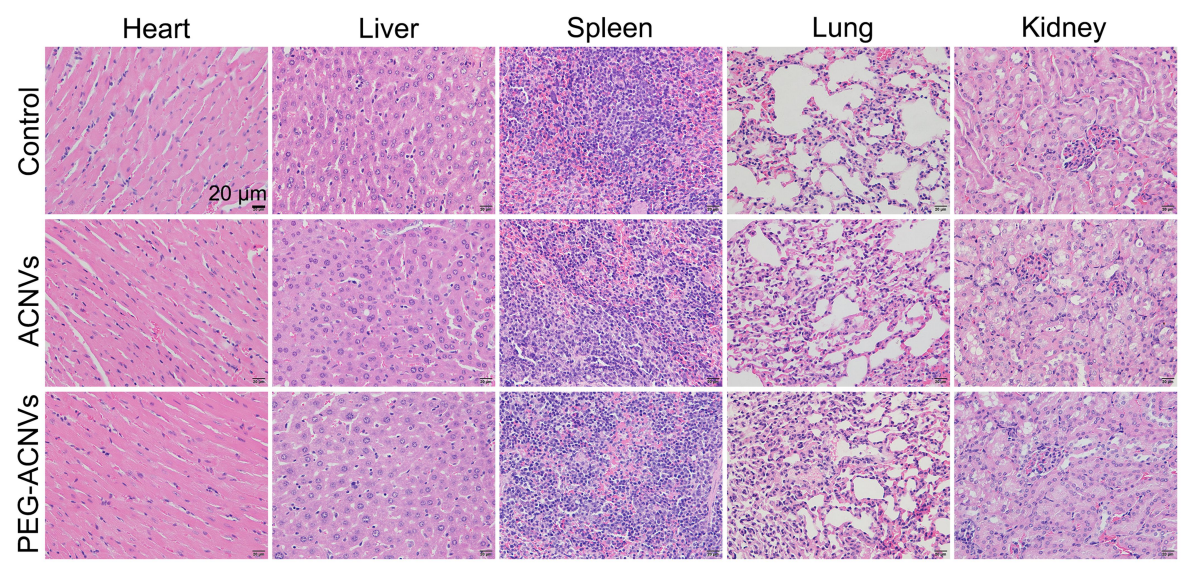

B
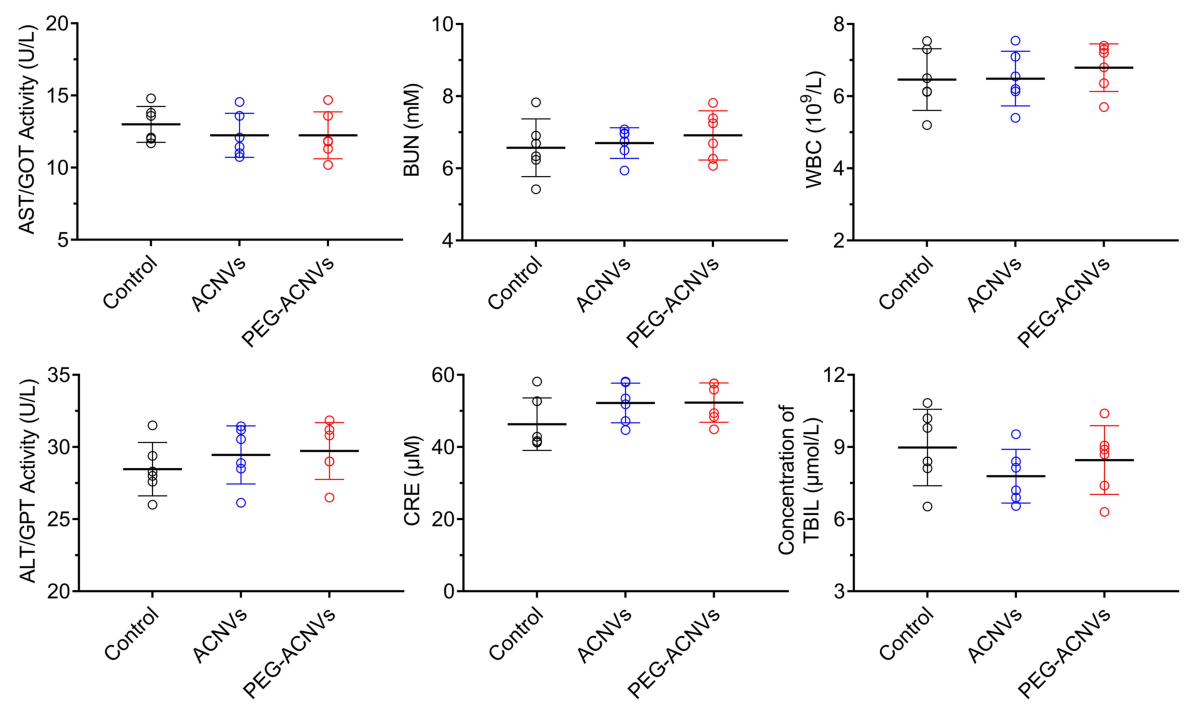

Figure 7 Safety evaluation of PEG-ACNVs. (A) H\&E staining of major tissues after treatment with ACNVs, PEG-ACNVs, and saline for 14 days, scale bar = $20 \mu$ m. (B) Serum biochemistry and routine blood test results (AST, ALT, BUN, CRE, WBC and T-Bil) of mice receiving different treatments ( $n=5$ ).

tumor targeting. Histopathological observations revealed that massive cancer cell remission occurred in the tumor tissues after receiving ACNVs and PEG-ACNVs. The higher efficiency of PEG-ACNVs group indicated that the inhibition of tumor growth could be partially attributed to the increased tumor accumulation and apoptosis effect (Figure $6 \mathrm{E}$ and $\mathrm{F}$ ). ${ }^{43}$ Moreover, neither obvious pathological abnormality in other organs (Figure 7A) nor difference in biochemistry parameters and blood routine was found (Figure 7B) during the study, indicating negligible systemic toxicity.

\section{Conclusion}

In this work, ACNVs were obtained by differential centrifugation and glucose gradient centrifugation. The optimized ACNVs had a regular cup-like nanostructure, and the particle size measured by TEM and NTA was about
$120 \mathrm{~nm}$. The composition analysis showed that ACNVs were mainly comprised of lipids, proteins, and nucleic acids. In vitro antitumor results showed that ACNVs could suppress the proliferation of tumors, induce cellular apoptosis and upregulate apoptosis-related factors in hepatocellular carcinoma cells, while little damage to the normal hepatocytes may be partially attributed to the different cellular uptake ability. In vivo distribution experiments showed that ACNVs could be cleared by MPS. Blocking SRs or PEG engineering could significantly improve blood circulation period and enhance the tumor-targeting ability of ACNVs. In vivo pharmacodynamic studies showed that PEGylated ACNVs could better inhibit tumor growth without significant side effects. In conclusion, ACNVs as one promising anticancer alternative offer the potential over the traditional extracts from A. cochinchinensis of 
satisfactory therapeutic benefits and better drug-like properties. This study could lay template for the development and utilization of other fresh edible and medicinal plants.

\section{Acknowledgments}

This work was supported by the National Natural Science Foundation of China (No. 81803456), the Young Elite Scientists Sponsorship Program by CAST (2017QNRC001), Key project at the central government level: The ability establishment of sustainable use for valuable Chinese medicine resources (2060302-1907-04), Natural Science Research of Jiangsu Higher Education Institutions of China (20KJA360004), Jiangsu Postgraduate Research \& Practice Innovation Program (KYCX20_1497, KYCX20_1498), Six Talent Peaks Project of Jiangsu Province (SWYY-057), and QingLan Project of Jiangsu Province of China.

\section{Disclosure}

The authors report no conflicts of interest in this work.

\section{References}

1. van Niel G, D'Angelo G, Raposo G. Shedding light on the cell biology of extracellular vesicles. Nat Rev Mol Cell Biol. 2018;19 (4):213-228. doi:10.1038/nrm.2017.125

2. Maas SLN, Breakefield XO, Weaver AM. Extracellular vesicles: unique intercellular delivery vehicles. Trends Cell Biol. 2017;27 (3):172-188. doi:10.1016/j.tcb.2016.11.003

3. Kalluri R, LeBleu VS. The biology, function, and biomedical applications of exosomes. Science. 2020;367(6478):1-15. doi:10.1126/ science.aau6977

4. Gyorgy B, Hung ME, Breakefield XO, Leonard JN. Therapeutic applications of extracellular vesicles: clinical promise and open questions. Annu Rev Pharmacol Toxicol. 2015;55(1):439-464. doi:10.1146/annurev-pharmtox-010814-124630

5. Cai Q, Qiao L, Wang M, et al. Plants send small RNAs in extracellular vesicles to fungal pathogen to silence virulence genes. Science. 2018;360(6393):1126-1129. doi:10.1126/science.aar4142

6. Petrovska BB. Historical review of medicinal plants' usage. Pharmacogn Rev. 2012;6(11):1-5. doi:10.4103/0973-7847.95849

7. Harvey AL, Edrada-Ebel R, Quinn RJ. The re-emergence of natural products for drug discovery in the genomics era. Nat Rev Drug Discov. 2015;14(2):111-129. doi:10.1038/nrd4510

8. Kong LY, Tan RX. Artemisinin, a miracle of traditional Chinese medicine. Nat Prod Rep. 2015;32(12):1617-1621. doi:10.1039/ c5np00133a

9. Fernandes Neto JM, Nadal E, Bosdriesz E, et al. Multiple low dose therapy as an effective strategy to treat EGFR inhibitor-resistant NSCLC tumours. Nat Commun. 2020;11(1):3157. doi:10.1038/ s41467-020-16952-9

10. Rome S. Biological properties of plant-derived extracellular vesicles. Food Funct. 2019;10(2):529-538. doi:10.1039/c8fo02295j

11. Zhang M, Viennois E, Prasad M, et al. Edible ginger-derived nanoparticles: a novel therapeutic approach for the prevention and treatment of inflammatory bowel disease and colitis-associated cancer. Biomaterials. 2016;101:321-340. doi:10.1016/j.biomaterials.2016. 06.018
12. Zhuang $\mathrm{X}$, Deng ZB, Mu J, et al. Ginger-derived nanoparticles protect against alcohol-induced liver damage. $J$ Extracell Vesicles. 2015;4(1):28713. doi:10.3402/jev.v4.28713

13. Cao M, Yan H, Han X, et al. Ginseng-derived nanoparticles alter macrophage polarization to inhibit melanoma growth. J Immunother Cancer. 2019;7(1):326. doi:10.1186/s40425-019-0817-4

14. Cheng W, Cheng Z, Xing D, Zhang M. Asparagus polysaccharide suppresses the migration, invasion, and angiogenesis of hepatocellular carcinoma cells partly by targeting the HIF-1alpha/VEGF signalling pathway in vitro. Evid Based Complement Alternat Med. 2019;2019:3769879. doi:10.1155/2019/3769879

15. Shen Y, Xu CL, Xuan WD, et al. A new furostanol saponin from Asparagus cochinchinensis. Arch Pharm Res. 2011;34 (10):1587-1591. doi:10.1007/s12272-011-1001-7

16. Chun JM, Cheon MS, Moon BC, Lee AY, Choo BK, Kim HK. Antitumor activity of the ethyl acetate fraction from Asparagus cochinchinensis in HepG2-xenografted nude mice. J Korean Soc Appl Biol Chem. 2011;54(4):538-543. doi:10.3839/jksabc.2011.082

17. Samad NB, Debnath T, Abul Hasnat M, et al. Phenolic contents, antioxidant and anti-inflammatory activities of Asparagus cochinchinensis (Loureiro) merrill. J Food Biochem. 2014;38(1):83-91. doi:10.1111/jfbc. 12028

18. Lei L, Chen Y, Ou L, Xu Y, Yu X. Aqueous root extract of Asparagus cochinchinensis (Lour.) Merr. has antioxidant activity in D-galactoseinduced aging mice. BMC Complement Altern Med. 2017;17(1):469. doi:10.1186/s12906-017-1975-x

19. Sun Q, Zhu L, Li Y, et al. A novel inulin-type fructan from Asparagus cochinchinensis and its beneficial impact on human intestinal microbiota. Carbohydr Polym. 2020;247:116761. doi:10.1016/j. carbpol.2020.116761

20. Deng Z, Rong Y, Teng Y, et al. Broccoli-derived nanoparticle inhibits mouse colitis by activating dendritic cell AMP-activated protein kinase. Mol Ther. 2017;25(7):1641-1654. doi:10.1016/j. ymthe.2017.01.025

21. Xiao S, Gao W, Chen QF, et al. Overexpression of arabidopsis acyl-CoA binding protein ACBP3 promotes starvation-induced and age-dependent leaf senescence. Plant Cell. 2010;22(5):1463-1482. doi:10.1105/tpc. 110.075333

22. Chen X, Zhou Y, Yu J. Exosome-like nanoparticles from ginger rhizomes inhibited NLRP3 inflammasome activation. Mol Pharm. 2019;16(6):2690-2699. doi:10.1021/acs.molpharmaceut.9b00246

23. Qiao H, Fang D, Zhang L, et al. Nanostructured peptidotoxins as natural pro-oxidants induced cancer cell death via amplification of oxidative stress. ACS Appl Mater Interfaces. 2018;10(5):4569-4581. doi:10.1021/acsami.7b18809

24. Yang M, Ding J, Feng X, et al. Scavenger receptor-mediated targeted treatment of collagen-induced arthritis by dextran sulfate-methotrexate prodrug. Theranostics. 2017;7(1):97-105. doi:10.7150/thno. 16844

25. Chen G, Huang AC, Zhang W, et al. Exosomal PD-L1 contributes to immunosuppression and is associated with anti-PD-1 response. Nature. 2018;560(7718):382-386. doi:10.1038/s41586-018-0392-8

26. Park M, Cheon MS, Kim SH, et al. Anticancer activity of asparagus cochinchinensis extract and fractions in HepG2 cells. J Korean Soc Appl Bio Chem. 2011;54(2):188-193. doi:10.3839/ jksabc.2011.031

27. Hong SJ, Dawson TM, Dawson VL. Nuclear and mitochondrial conversations in cell death: PARP-1 and AIF signaling. Trends Pharmacol Sci. 2004;25(5):259-264. doi:10.1016/j.tips.2004.03.005

28. Mu J, Zhuang X, Wang Q, et al. Interspecies communication between plant and mouse gut host cells through edible plant derived exosome-like nanoparticles. Mol Nutr Food Res. 2014;58 (7):1561-1573. doi:10.1002/mnfr.201300729

29. Xiao J, Feng S, Wang X, et al. Identification of exosome-like nanoparticle-derived microRNAs from 11 edible fruits and vegetables. PeerJ. 2018;6:e5186. doi:10.7717/peerj.5186 
30. Huang B, Song BL, Xu C. Cholesterol metabolism in cancer: mechanisms and therapeutic opportunities. Nat Metab. 2020;2 (2):132-141. doi:10.1038/s42255-020-0174-0

31. Zhang L, Bennett WF, Zheng T, et al. Effect of cholesterol on cellular uptake of cancer drugs pirarubicin and ellipticine. J Phys Chem B. 2016;120(12):3148-3156. doi:10.1021/acs.jpcb.5b12337

32. Wu H, Yu M, Miao Y, et al. Cholesterol-tuned liposomal membrane rigidity directs tumor penetration and anti-tumor effect. Acta Pharm Sin B. 2019;9(4):858-870. doi:10.1016/j.apsb.2019.02.010

33. Hoshino A, Costa-Silva B, Shen TL, et al. Tumour exosome integrins determine organotropic metastasis. Nature. 2015;527(7578):329-335. doi: $10.1038 /$ nature 15756

34. Wang Q, Zhuang X, Mu J, et al. Delivery of therapeutic agents by nanoparticles made of grapefruit-derived lipids. Nat Commun. 2013;4 (1):1867. doi:10.1038/ncomms2886

35. Donahue ND, Acar H, Wilhelm S. Concepts of nanoparticle cellular uptake, intracellular trafficking, and kinetics in nanomedicine. $A d v$ Drug Deliv Rev. 2019;143:68-96. doi:10.1016/j.addr.2019.04.008

36. Tsoi KM, MacParland SA, Ma XZ, et al. Mechanism of hard-nanomaterial clearance by the liver. Nat Mater. 2016;15 (11):1212-1221. doi:10.1038/nmat4718

37. Blanco E, Shen H, Ferrari M. Principles of nanoparticle design for overcoming biological barriers to drug delivery. Nat Biotechnol. 2015;33(9):941-951. doi:10.1038/nbt.3330
38. Matsumoto A, Takahashi Y, Chang HY, et al. Blood concentrations of small extracellular vesicles are determined by a balance between abundant secretion and rapid clearance. J Extracell Vesicles. 2020;9 (1):1696517. doi:10.1080/20013078.2019.1696517

39. Watson DC, Bayik D, Srivatsan A, et al. Efficient production and enhanced tumor delivery of engineered extracellular vesicles. Biomaterials. 2016;105:195-205. doi:10.1016/j. biomaterials.2016.07.003

40. Furumoto K, Nagayama S, Ogawara K, et al. Hepatic uptake of negatively charged particles in rats: possible involvement of serum proteins in recognition by scavenger receptor. $J$ Control Release. 2004;97(1):133-141. doi:10.1016/j.jconrel.2004.03.004

41. Maeda H, Nakamura H, Fang J. The EPR effect for macromolecular drug delivery to solid tumors: improvement of tumor uptake, lowering of systemic toxicity, and distinct tumor imaging in vivo. $A d v$ Drug Deliv Rev. 2013;65(1):71-79. doi:10.1016/j.addr.2012.10.002

42. Suk JS, Xu Q, Kim N, Hanes J, Ensign LM. PEGylation as a strategy for improving nanoparticle-based drug and gene delivery. Adv Drug Deliv Rev. 2016;99(PtA):28-51. doi:10.1016/j.addr.2015.09.012

43. Jiang T, Shen S, Wang T, Li M, He B, Mo R. A substrate-selective enzyme-catalysis assembly strategy for oligopeptide hydrogel-assisted combinatorial protein delivery. Nano Lett. 2017;17(12):7447-7454. doi:10.1021/acs.nanolett.7b03371
International Journal of Nanomedicine

\section{Publish your work in this journal}

The International Journal of Nanomedicine is an international, peerreviewed journal focusing on the application of nanotechnology in diagnostics, therapeutics, and drug delivery systems throughout the biomedical field. This journal is indexed on PubMed Central, MedLine, CAS, SciSearch ${ }^{\mathbb{}}$, Current Contents ${ }^{\mathbb{R}} /$ Clinical Medicine, $^{2}$

\section{Dovepress}

Journal Citation Reports/Science Edition, EMBase, Scopus and the Elsevier Bibliographic databases. The manuscript management system is completely online and includes a very quick and fair peer-review system, which is all easy to use. Visit http://www.dovepress.com/ testimonials.php to read real quotes from published authors. 